\title{
Function of Big Data Processing on E-commerce Development under Cloud Computing Environment
}

\author{
Xianwei Feng \\ Jiangsu Institute of Commerce, Nanjing, 211168, China
}

\begin{abstract}
Keywords: Cloud computing environment; Big Data processing; E-Commerce development; Specific function
\end{abstract}

\begin{abstract}
E-commerce highlights overall tendency of virtualization and shows transparency. With the help of efficiency advantage, it rapidly extends data processing and data transmission under network path, and occupies a main market. Cloud computing is classified into a novel mode and shows leap-frog development by depending network. Seeing from currants status, information loaded by network progressively increases, and numerical treatment under traditional path is difficult to comply with requirements. In cloud computing environment, it is required to analyze the influence of big data analysis and processing on e-commerce development and propose application strategies.
\end{abstract}

\section{Introduction}

With network expansion, network users reach hundred million. Progressive increase of e-commerce is evolving orderly. Under cloud computing status, numerical values left by enterprises are too many and explosive growth stands out. All kinds of shopping in ordinary days expand numerical values within the category of cloud computing. Such progressive increase in data also increases the pressure of network facilities. it is very hard to manage orderly if traditional facilities are adopted. If cloud computing is applied, this difficulty may be solved. Specific processing steps of big data promote business progress and accelerates evolution speed of e-commerce.

\section{Analysis of operating mechanism}

Common cloud computing extends initial parallel computation, grid computing and specific distribution operation. It creates processing and operation under large-scale situation by depending on microcomputer. The processing program selected can be automatically subdivided into multiple detailed subprograms. Such subprograms are given to network system for search. After operation and analysis, such subprograms are returned to users again. Under such mode, it is unnecessary to identify the memory property, load and operation feature specifically owned by the terminal. Cloud computing makes convenient to exchange resources and share resources. Cloud computing expands application effect in network architecture, reduce overall spending and boost operation efficiency.

Commercial mode subdivided from cloud computing contains platform service, basic framework service and kit software service. Such packaged service is closely related to network framework. Operating capability is set up independent commodity. It can be exchanged by depending on network. The overall framework constructed by cloud computing contains data center. Data are exchanged, and resources are retained on the basis if such center. Consumption is conducted properly as per the set index. Users can interact and expend in each time bucket, which adds convenience.

\section{E-commerce features}

In big data status, overall data generated in ordinary days are big. According to speculation, in future time bucket, data will also present the law of explosive progressive increase. E-commerce website tallies with this development tendency. To be more specific, e-commerce website will experience three development time buckets. 
In the first time bucket, e-commerce website puts particular emphasis on accumulating users. The peculiar profit path of the website is to expand the number of users, collect essential money and create website advertisement, in the second time bucket, e-commerce website puts particular emphasis on accumulating total sales volume so as to expand profit. In this time bucket, enterprises depend on media and network to put too many advertisements for expanding publicity and adding extra brand value. In the third time bucket, data appreciate rapidly. Commercial enterprises depend on incremental users to accumulate transaction data and gain big data. Business process covers initial numerical value collection, follow-up numerical value analysis, integration and digging. For e-commerce, its prominent individuality should be mainly evaluated to achieve accurate operation. The big data accumulated may expand the market and extend enterprise prospect in the future time bucket.

\section{Recognition of the relationship between the two}

\section{Novel commercial connotation}

Under big data, peculiar connotation of e-commerce integrates dual connotation: broad and narrow. In a broad sense, it contains e-transaction in ordinary days and exchanges via network. For conventional activities, it refers to individual activity within enterprise category. In a narrow sense, e-commerce purely refers to conventional trade in ordinary days. E-commerce is integrated within economy, connected to a complete entirety by depending on network and runs through multiple links. Such integration reduces inherent distance of each other. Owning information technology is the foundation of mutual connection. Digital economy in e-commerce framework contains network data. Such prominent advantage can recognize economic status within macro and micro category and maintain economic stability.

\section{Commercial relations under big data}

The operation of e-commerce cannot break away from microcomputer support. Seeing from operating system, clearness feature of cloud computing is networking. Such integrated network contains set topology framework, the set of servers supplies the optimal relevance operation. Under network environment, without the microcomputer, network resources retained can be still applied to exchange information and share smoothly.

Cloud computing promotes original security hierarchy. Such security feature contains storage security and conventional operation security. Diversified approaches are adopted to guarantee data security. This is because e-commerce associated by big data depends on a wider range of network environment. Besides, trade secret is also closely related to users' interest. Only when trade secret is properly kept can development be promoted. E-commerce confidentiality is regarded as the foundation of origin. After the trade ends, it is required to continue to keep secret, and the rights and interests cannot be impaired. Thus, it is required to keep prudent and maintain one's rights and interests in the trade.

Under big data status, it is also required to pay attention to adopt appropriate encryption technique, control security by encryption and avoid network threat. Besides, encryption technique should be added to maintain flexibility and security in network architecture. At the same time, due protection should be provided to solve several difficulties in daily transactions. Cloud computing creates distributed overall framework. Software may be deemed as specific immediate service which is supplied for users in the section. The specific system of cloud computing can eliminate potential virus, bring convenience for sharing in each time bucket and expand network resources. 


\section{Analysis of specific functions}

\section{Accurate analysis of data}

E-commerce process contains mass data. Real-time analysis is set for such data. Such respect is set to the emphasis in competition category. The unique value of big data is to prudently identify and analyze diversified data, intensify information analysis in the maximum category, accurately dig data and then set deep-level analysis steps. In initial analysis, metadata should be registered. Double technique is adopted to construct the overall framework on JKTA foundation. The nodes managed contain dual-level ring structure, also called cloud structure. Initial information exists in the subdivided nodes. Each node is interacted and keeps respective initial routing tables. In follow-up operation, with progressive increase and progressive decrease of nodes, special framework of P2P is kept intact, and correct resources are retained. To discover resources, it is required to consider subschema under overall framework and choose information unit. Diversified association patterns should be matched with each other and contain semantic difference. Services should be set according to users' requirements. In the query steps, relevant requests in the overall category are extended on the basis of the field. Such requests are subdivided into matched subschema. Based on the accurate information collected, it is required to check and write again to generate appropriate execution plans. Integrated data steps contain a set of plans required by sub-query. Under transmission path, it is required to integrate such data within the minimum category and optimize each site for integration realization. For copy management, there should be multiple backups which contain diversified nodes. The database constructed should be also backed up. Proper backup maintains inherent completeness of metadata and boosts examination effect. All kinds of subdivided sites should be checked in follow-up execution.

The database retains data within e-commerce category. For the overall situation, the data should be kept properly so as to execute dispatching. The data in distributed framework should be properly processed. The integration result may be analyzed to improve visualization. As per the real request submitted, the result is gained through integration. Big data are used to analyze e-commerce marketing steps, which can obtain prominent effects. This is because cloud computing searches and digs diversified data in a short time bucket, retains such information and then properly analyzes the data. This provides the necessary reference for marketing decision to analyze marketing groups, customers' preference and tendency, and reduce marketing deviation.

\section{Flexible processing}

Commercial system should accelerate original processing speed and adopt flexible processing. Such processing should be set to the fundamental objective. Flexible processing can help to cope with booming visit under emergency, handle mass orders and browse customers' requirements. Meanwhile, according to progressively increasing real requirements and extended volume of business, inherent servers should be expanded. For relevant data, a set of storage accessories should be added.

Special novel platforms of cloud storage should be constructed on the basis of cloud computing. Such platforms highlight unique advantages and can store mass data and adapt operation resources under large-scale situation. The hierarchy promoted contains numerical value processing in PB hierarchy. Enterprises do not need to add new hardware, which reduces expenditure. With the need of matching hardware, a set of system can be deployed rapidly. Meanwhile, guaranteed elastic expansion adds the ability of deploying and controlling within the maximum hierarchy and optimized integrated resources. For example, in 11.1 Festival, the sales volume of Tmall and Taobao rapidly increased to 5.8 billion. Such large-scale sales cannot be separated from the set good countermeasure. It is thus clear that, under big data status, e-commerce extension is closely related to the platform created by cloud computing. It is required to boost operation efficiency and enhance processing flexibility. 


\section{Checking and retrieving information}

E-commerce process can lay emphasis on expanding original merchandise categories and boost competitive strength of overall framework. However, a large number of commodities are classified in subdivided system. The database should be provided to add original checking property and retrieving property. Seeing from current situation, network exploration and analysis of database fail to be deepened and are still in progress. Related surveys are also not many. If cloud computing is adopted, information checking and information retrieval under overall situation may be provided.

Recognize user difference and set matched search according to individualities. The returned numerical value should be accurate, and precise probability should improve. In another level, information may also be pushed. For hot information checked, new retrieval should be provided. To a large extent, it tallies with uncertain alteration network. Dynamic numerical values in multiple fields should be integrated to construct grid system matched with the database. It is necessary to effectively manage database, gain accurate information and boost checking effect in ordinary days.

\section{New path construction}

\section{Expand full-process commerce}

Cloud computing under global framework is applied to construct supply chain cloud under the support of big data. With such system, e-commerce extends new full-process mode. The big data provide the optimal, most reliable and stable operation process. This lowers the demand of terminal matching, deploys resources easily, shares data and exchanges data. As long as the demand is owned, multiple space services may be supplied. Such unique advantage fuses diversified existing resources, which lays a solid foundation for providing superior services.

The suppliers associated with cloud computing are covered in an integrated chain. Hence, the industry chain is constructed. The new service system contains data services under gold abacus and large-scale framework. The new services of supply chain cover the whole process, and can be widely adopted.

\section{Create mobile cloud}

New-type integrated commerce contains cloud computing under mobile path and fuses triple network. Under such tendency, new services with mobility feature alter old mode. Cloud computing with a huge system highlights the optimal distribution feature. It promotes original information ability and improves operation efficiency. As per allocation based on the demands, it breaks several shortcomings under traditional path, brings new terminal and breaks through bottleneck and obstacle of long time bucket. With the new processing specially owned by big data, the security will not be worried. Guarantee may be added for ordinary data transmission and diversified processing under conventional situation. Services can be accepted in each time bucket. Users are fused in the platform.

Big data novel mode of mobile cloud solves the problem of mobile terminal, brings convenience for data sharing and promotes task execution. Initial hardware requirements are eliminated for mobile phone terminal and other matched terminals. Simple equipment can be connected with such cloud terminal to gain information. For e-commerce itself, it is unnecessary to provide the mobile platform under complex framework. As long as the lease is applied for, services can be gained and the property can be expanded quickly. Such shortcut fuses multi-hierarchy new properties and creates brand-new space.

\section{Improve actual effect of commerce}

Arrange new system and check control in the whole process. For logistics system, we can create complete supply chain, online return and order, check accounts and dealers' inventory, control cost orderly and construct new websites. Website maintenance in follow-up time bucket can be done by commercial team. Special teams are matched to supply the optimal and most reliable guarantee. 
After the new scheme is applied, ordering speed is accelerated and the cost is also reduced. Besides, processing time bucket decreases by $78 \%$. Customers are very satisfied, and business volume increase progressively. Thus, we can know that cloud computing of big data creates chain value in the maximum category.

\section{Conclusion}

Under big data environment, e-commerce shows integrated development trend. Thus, information collection in each time bucket, follow-up information analysis and storage security should be considered. Cloud computing extends network resources and own the scalability feature. User subject should be taken into account as per real demand. Only in this way, new commerce driven by demands may be created. In future development, cloud computing highlights the leading position and facilitates era development.

\section{Acknowledgments}

This paper is one of research results of key topic of Jiangsu Institute of Commerce - Study on Precision Marketing Personnel Cultivation Mode of Vocational Colleges under Big Data Entrainment. No.: JSJM1414

\section{References}

[1] Kang Li, Study on e-commerce security and countermeasures under cloud computing environment. Management \& Technology of SME (periodical appearing in the first ten days), 2013 (02): 283-284.

[2] Quan Shifeng, Function of big data processing on e-commerce development under cloud computing environment. Computer Knowledge and Technology, 2013 (20): 4762-4763+4770.

[3] Zhang Xin, Analysis of e-commerce development under big data environment. China Science and Technology Information, 2014 (23): 197-200.

[4] Zhou Benhai, On influence of big data on e-commerce under cloud computing environment. Economic Research Guide, 2015 (07): 201-202.

[5] Yang Fang, Influence of cloud computing on e-commerce under big data environment. Silicon Valley, 2014 (06): 180-181. 